УДК 539.3

\title{
РАСЧЁТ СТАТИЧЕСКИ НЕОПРЕДЕЛИМОЙ БАЛКИ МЕТОДОМ КОНЕЧНЫХ РАЗНОСТЕЙ
}

\author{
Нагоев Айдемир Муратович \\ Аккулова Мадина Муслимовна \\ студенты \\ Научный руководитель: Культербаев Хусен Пшимурзович \\ д.т.н., профессор \\ СКФУ «Северо-Кавказский центр \\ математических исследований»
}

\begin{abstract}
Аннотация: Рассматриваются статически неопределимые балки, нагруженные непрерывно распределённой поперечной нагрузкой. Используется математическая модель задачи, состоящая из обыкновенного дифференциального уравнения и граничных условий, заданных в континуальной области. $\mathrm{C}$ помощью метода конечных разностей осуществляется переход в сеточную область. Разработан алгоритм вычислений численного метода и показан пример его применения. По полученным результатам сделаны практические выводы.

Ключевые слова: Дифференциальное уравнение; краевые условия; сеточная область; метод конечных разностей; система алгебраических уравнений; вычислительный комплекс Matlab.
\end{abstract}

\section{CALCULATION OF A STATICALLY UNDETERMINABLE BEAM BY THE FINITE DIFFERENCE METHOD}

\section{Nagoev Aydemir Muratovich Akkulova Madina Muslimovna Kulterbaev Khusen Pshimurzovich}

\begin{abstract}
Statically indeterminate beams loaded with a continuously distributed transverse load are considered. A mathematical model of the problem is used, which consists of an ordinary differential equation and boundary conditions specified in the continual domain. Using the finite difference method, the transition to
\end{abstract}


the grid area is carried out. An algorithm for calculating the numerical method is developed and an example of its application is shown. Based on the results obtained, practical conclusions are drawn.

Key words: Differential equation; boundary conditions; mesh area; finite difference method; system of algebraic equations; computing complex Matlab.

\section{Введение}

Среди строительных и машиностроительных конструкций балки являются самыми распространенными. Их расчёт и проектирование изучаются во многих учебных дисциплинах: сопротивление материалов, механика конструкций, строительная механика, динамика и устойчивость сооружений, детали машин и приборов. По этой причине изучение балок, начатое ещё в XIX веке, продолжается до настоящего времени и имеет уже обширную библиографию в виде научных монографий, учебников и многочисленных статей [1 - 4]. В начальный период исследование балок проводилось лишь аналитическими методами путём решения дифференциальных уравнений.

В настоящее время существует большое разнообразие типов балок по их материалу (деревянные, металлические, железобетонные), типам опор, формам поперечного сечения, по методам их расчёта (статически определимые, статически неопределимые). Наиболее сложными являются расчёты статически неопределимых балок. Аналитические методы в таких случаях оказываются громоздкими и не универсальными. Математическая модель расчёта является двухэтапным: сначала раскрывается статическая неопределенность задачи, затем применяются различные алгоритмы аналитических решений: методы непосредственного интегрирования, начальных параметров, формулы Мора, правило Верещагина, методы сил и перемещений и т. д.

В связи с появлением в последние десятилетия мощных вычислительных машин, персональных компьютеров, программных комплексов для расчёта и проектирования механических конструкций широкое распространение получили численные методы расчёта. К ним относятся метод конечных элементов и метод конечных разностей. Преимущества этих методов особенно заметны, когда балки являются статически неопределимыми и их расчёт классическими методами является многоэтапным. Для решения несложных задач целесообразным представляется применение численного метода конечных разностей [6 - 9] в среде Matlab. 


\section{1. Математическая модель задачи}

Изогнутая ось балки описывается дифференциальным уравнением четвёртого порядка

$$
\operatorname{EJv}^{\prime \prime}(\mathrm{x})=\mathrm{q}(\mathrm{x}) \text {. }
$$

Преобразуем его к более удобному виду

$$
\mathrm{v}^{\prime \prime \prime}(\mathrm{x})=\mathrm{p}(\mathrm{x}), \quad \mathrm{p}(\mathrm{x})=\frac{\mathrm{q}(\mathrm{x})}{\mathrm{b}}, \quad \mathrm{b}=\mathrm{EJ} .
$$

К уравнению (1) присоединяются граничные условия. Примем их для расчётной схемы по рис. 1. Левый конец балки защемлён. Поэтому здесь прогиб и угол поворота равны нулю

$$
\mathrm{v}(0)=0, \quad \mathrm{v}^{\prime}(0)=0 .
$$

На правом конце шарнирная опора и ей соответствуют краевые условия

$$
\mathrm{v}(l)=0, \quad \mathrm{v}^{\prime \prime}(l)=0 .
$$

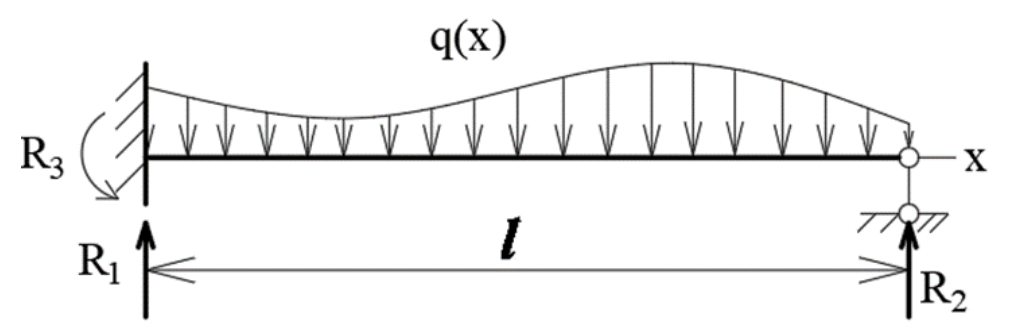

Рис. 1.

Область непрерывного изменения аргумента $[0, l]$ заменим областью дискретного изменения

$$
\mathrm{L}_{\mathrm{h}}=\left\{\mathrm{x}_{\mathrm{i}}=(\mathrm{i}-1) \mathrm{h}, \mathrm{i}=1,2, \ldots, \mathrm{n}\right\} \text {. }
$$

Множество точек с номерами і называется сеткой, а сами точки узлами сетки. Далее вместо функции непрерывного аргумента $\mathrm{v}=\mathrm{v}(\mathrm{z})$ будет отыскиваться сеточная функция $\mathrm{v}_{\mathrm{i}} \approx \mathrm{v}\left(z_{\mathrm{i}}\right)$.

\section{2. Алгоритм вычислений}

Производные и значения функции $\mathrm{v}$ в задаче (1) - (3) заменим конечноразностными соотношениями и значениями дискретной функции на пятиточечном шаблоне сетки (рис. 2): 


$$
\begin{gathered}
\mathrm{v}^{\prime \prime \prime}\left(\mathrm{x}_{\mathrm{i}}\right) \approx \frac{1}{\mathrm{~h}^{4}}\left(\mathrm{v}_{\mathrm{i}-2}-4 \mathrm{v}_{\mathrm{i}-1}+6 \mathrm{v}_{\mathrm{i}}-4 \mathrm{v}_{\mathrm{i}+1}+\mathrm{v}_{\mathrm{i}+2}\right), \quad \mathrm{v}(0)=\mathrm{v}_{1}=0, \\
\mathrm{v}^{\prime}(0) \approx \frac{1}{2 \mathrm{~h}}\left(-3 \mathrm{v}_{1}+4 \mathrm{v}_{2}-\mathrm{v}_{3}\right)=0, \quad \mathrm{v}(l)=\mathrm{v}_{\mathrm{n}}, \\
\mathrm{v}^{\prime \prime}(l) \approx \mathrm{v}_{\mathrm{n}}^{\prime \prime}=\frac{1}{\mathrm{~h}^{2}}\left(-\mathrm{v}_{\mathrm{n}-3}+4 \mathrm{v}_{\mathrm{n}-2}-5 \mathrm{v}_{\mathrm{n}-1}+2 \mathrm{v}_{\mathrm{n}}\right)=0 .
\end{gathered}
$$

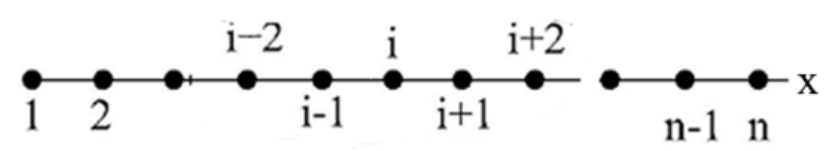

Рис. 2.

Подстановка (4) в задачу (1) - (3) и элементарные преобразования приводят к конечноразностной схеме:

$$
\begin{aligned}
& \mathrm{v}_{\mathrm{i}-2}-4 \mathrm{v}_{\mathrm{i}-1}+6 \mathrm{v}_{\mathrm{i}}-4 \mathrm{v}_{\mathrm{i}+1}+\mathrm{v}_{\mathrm{i}+2}=\mathrm{p}_{\mathrm{i}}, \quad \mathrm{p}_{\mathrm{i}}=\mathrm{q}_{\mathrm{i}} \mathrm{h}^{4} / \mathrm{b}, \quad \mathrm{i}=3,4, \ldots, \mathrm{n}-2 ; \\
& \mathrm{v}_{1}=0, \quad-3 \mathrm{v}_{1}+4 \mathrm{v}_{2}-\mathrm{v}_{3}=0 ; \quad-\mathrm{v}_{\mathrm{n}-3}+4 \mathrm{v}_{\mathrm{n}-2}-5 \mathrm{v}_{\mathrm{n}-1}+2 \mathrm{v}_{\mathrm{n}}=0 \quad \mathrm{v}_{\mathrm{n}}=0 .
\end{aligned}
$$

Запишем её как систему алгебраических уравнений относительно вектора v в матрично-векторной форме

$$
\mathrm{Av}=\mathrm{p},
$$

где

$$
\begin{aligned}
& \mathrm{A}=\left(\begin{array}{cccccccccccccc}
1 & & & & & & \vdots & & & & & & \\
-3 & 4 & -1 & & & & \vdots & & & & & & \\
1 & -4 & 6 & -4 & 1 & & \vdots & & & & & & \\
& 1 & -4 & 6 & -4 & 1 & \vdots & & & & & & \\
\cdots & \cdots & \cdots & \ldots & \cdots & \cdots & \vdots & \cdots & \ldots & \cdots & \cdots & \ldots & \cdots \\
& & & & & & \vdots & 1 & -4 & 6 & -4 & 1 & \\
& & & & & & \vdots & & 1 & -4 & 6 & -4 & 1 \\
& & & & & & \vdots & & & -1 & 4 & -5 & 2 \\
& & & & & & \vdots & & & & & & 1
\end{array}\right), \\
& \mathrm{p}=\left\{0,0, \mathrm{p}_{3}, \mathrm{p}_{4}, \ldots, \mathrm{p}_{\mathrm{n}-3}, \mathrm{p}_{\mathrm{n}-2}, 0,0\right\}^{\mathrm{T}}, \quad \mathrm{p}_{\mathrm{i}}=\mathrm{q}_{\mathrm{i}} \mathrm{h}^{4} / \mathrm{b} . \quad \mathrm{i}=3,4, \ldots \mathrm{n}-2 .
\end{aligned}
$$

Нулевые элементы матрицы не показаны, значок $\mathrm{T}$ в индексе соответствует операции транспонирования вектора.

Пример. Дана стальная балки из двутавра №18 (рис. 1) с параметрами: длина пролёта $l=6 \mathrm{M}$, распределенная нагрузка $\mathrm{q}(\mathrm{x})=\mathrm{q}_{0}+\mathrm{q}_{1} \sin (\pi \mathrm{x} / l)$, $\mathrm{q}_{0}=3 \kappa \mathrm{H} / \mathrm{M}, \mathrm{q}_{1}=10 \kappa \mathrm{H} / \mathrm{M}$. 
Требуется: построить эпюры изгибающих моментов и определить прогибы изогнутой оси балки $\mathrm{v}_{\mathrm{i}}, \mathrm{i}=1,2, \ldots, \mathrm{n}$.

Система уравнений (5) решается на компьютере с помощью подпрограммы вычислительного комплекса Matlab, в результате чего становится известным вектор прогибов v. По нему затем вычисляются изгибающие моменты М (x) в сечениях

$$
\mathrm{M}=\mathrm{bv}^{\prime \prime} \text {. }
$$

В переходе на конечноразностное представление во внутрисеточных узлах они принимают вид

$$
\mathrm{M}_{\mathrm{i}}=\frac{\mathrm{b}}{\mathrm{h}^{2}}\left(\mathrm{v}_{\mathrm{i}-1}-2 \mathrm{v}_{\mathrm{i}}+\mathrm{v}_{\mathrm{i}+1}\right), \quad \mathrm{i}=3,4, \ldots, \mathrm{n}-2 .
$$

На левом конце в точках $\mathrm{i}=1,2$ изгибающие моменты представляются в виде

$$
\mathrm{M}_{1}=\frac{\mathrm{b}}{\mathrm{h}^{2}}\left(2 \mathrm{v}_{1}-5 \mathrm{v}_{2}+4 \mathrm{v}_{3}-\mathrm{v}_{4}\right), \quad \mathrm{M}_{2}=\frac{\mathrm{b}}{\mathrm{h}^{2}}\left(\mathrm{v}_{1}-\mathrm{v}_{2}+\mathrm{v}_{3}\right) .
$$

Аналогично на правом конце

$$
M_{n}=\frac{b}{h^{2}}\left(v_{n-3}+4 v_{n-2}-5 v_{n-1}+2 v_{n}\right), \quad M_{n-1}=\frac{b}{h^{2}}\left(v_{n-2}-2 v_{n-1}+v_{n}\right) .
$$

Результаты, полученные компьютером, показаны на рис. 3 - 5.

Распределённая нагрузка q

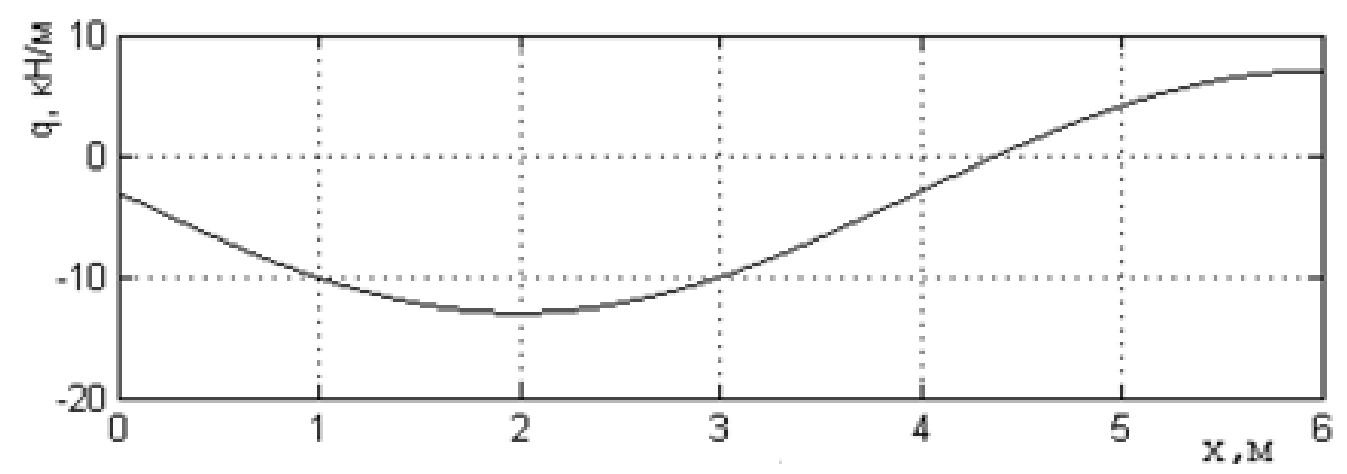

Рис. 3.

Функция прогибов v 


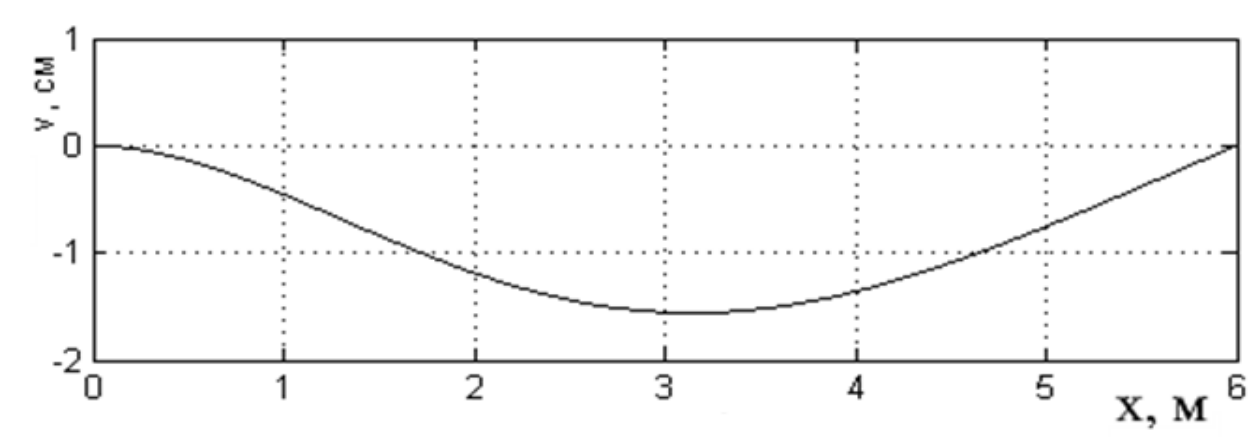

Рис. 4.

Изгибающие моменты М

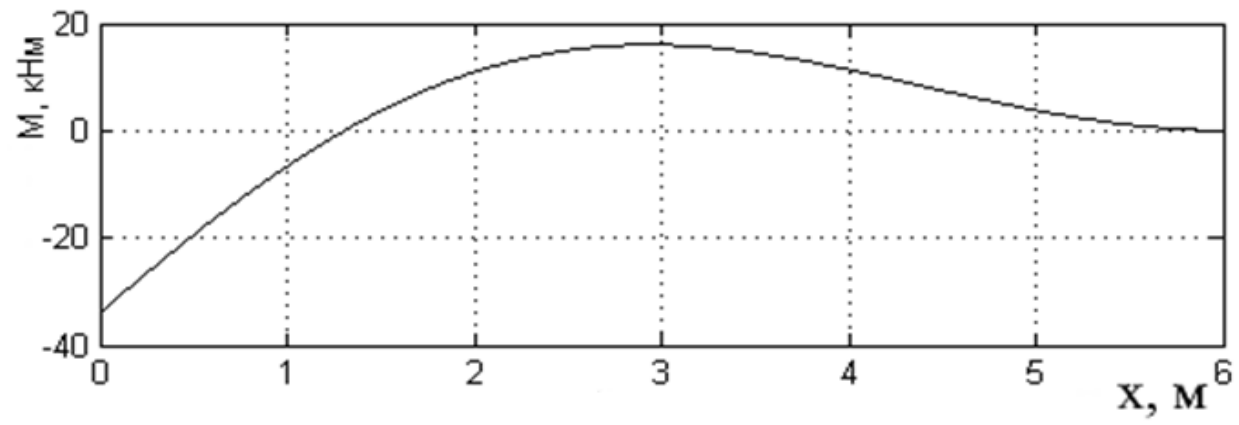

Рис. 5.

Эти результаты дают возможности для решения вопросов о прочности и жёсткости рассмотренной балки в соответствии с нормативными требованиями.

\section{Выводы}

1. Применение метода конечных разностей в сочетании с компьютерными технологиями приводит к значительному упрощению и сокращению расчётов статически неопределимых задач.

2. Метод конечных разностей обладает универсальностью и простотой адаптации к решению сложных задач при расчёте балок.

\section{Список литературы}

1. Феодосьев В.И. Сопротивление материалов. -М.: Изд. МГТУ им. Н.Э.Баумана, 2003. -592 с.

2. Атаров Н.М. Сопротивление материалов в примерах и задачах: Учебное пособие. -М.: ИНФРА-М, 2013. - 407 с. 
3. Антуфьев В.А., Горшков А.Г. и др. Сборник задач по сопротивлению материалов с теорией и примерами / Под ред. А.Г. Горшкова, Д.В. Тарлаковского. Учебное пособие. Для вузов._М.Ж ФИЗМАТЛИТ, 2003. -632 с.

4. Доннелл Л.Г. Балки, пластины и оболочки. -М.: Наука, 1982. 175 с.

5. Самарский А.А., Гулин А.В. Численные методы. М.: Наука, Гл. ред. физ.-мат. лит.,1989. - 432 с.

6. Варвак П.М., Варвак Л.П. Метод сеток в задачах расчёта строительных конструкций. М.: Стройиздат, 1977. 154 с.

7. Вержбицкий В.М. Основы численных методов. М.: Высшая школа, 2002. $840 \mathrm{c}$.

8. Ильин В.П., Карпов В.В., Масленников А.М. Численные методы решения задач строительной механики. - М.: Изд-во АСВ; СПб.: СПбГАСУ, 2005. -425 c.

9. Караманский Т.Д. Численные методы строительной механики. -М.: Стройиздат, 1981. -436 с.

(C) А.М. Нагоев, М.М. Аккулова, 2021 